\title{
SENTINEL LYMPH NODE BIOPSY AS GUIDANCE FOR CENTRAL NECK DISSECTION IN PATIENTS WITH PAPILLARY THYROID CARCINOMA
}

\author{
Ayman Abd-Allah Abd-Rabu, Mahmoud Saad Farahat, Haitham Mostafa Elmaleh, \\ Diaa Abdo Abdelaziz Bakr*
}

Department of General Surgery
Department, Faculty of
Medicine, Ain Shams
University , Cairo, Egypt
Corresponds:

Diaa Abdo Abdelaziz Bakr e.male:

Diaa_bakr2002@yahoo.com Tel.: 01006383143

Received: 20/2/2019

Accepted: 26/3/2019

\begin{abstract}
Background: Occult lymph node metastasis of papillary thyroid carcinoma (PTC) can be detected by sentinel lymph node (SLN) biopsy.
\end{abstract}

Aim of the work: Our objective in this study was to evaluate the accuracy of using sentinel lymph node biopsy as a marker to identify occult metastases early.

Patients and methods: The present study was a prospective study that was conducted in Ain Shams University Hospitals in Egypt, and included sixty (60) patients who had papillary thyroid carcinoma. The patients underwent sentinel lymph node biopsy with total thyroidectomy during the period between 1st of December 2016 and 1st of December 2018.

Results: The study included 60 patients with papillary thyroid carcinoma, 16 males and 44 females. Size of nodules ranged from 0.5 $4 \mathrm{~cm}$ and more nodules were confined to the left lobe (63.3\%). SLNs were identified in 44 of 60 malignant cases (73.3\%). 38 of identified SLN were positive for metastasis and 22 were negative while non SLN were positive in 31/60 and negative for 29/60. Thus, the sensitivity, specificity, negative predictive values, positive predictive values and accuracy of SLN biopsy were 86.7\%, 100\%, 72,7\%, 100\% and 73.3\%, respectively. Post-operative complications included temporary symptomatic hypocalcaemia in 3 patients and temporary recurrent laryngeal nerve injury in 2 patients then recovered.

Conclusion: SLN biopsy for patients with papillary thyroid carcinoma and negative LNs clinically and radiologically is an accurate and noninvasive means to identify subclinical lymph node metastasis.

Key words: Sentinel lymph node, differentiated thyroid carcinoma, zone 6 LN dissection, central neck dissection, STN.

\section{INTRODUCTION:}

Well differentiated papillary thyroid carcinoma (PTC) has a high propensity to spread to regional lymph nodes, with a frequency as high as $82 \%$ in patients with clinically negative lymph nodes $(\mathrm{cN} 0)^{(1)}$.
Lymphatic metastasis from differentiated PTC occurs in a stepwise fashion: first to the lymph nodes in the tracheoesophageal groove; and, subsequently, to lymph nodes in the jugular chain, including the supraclavicular fossa. Patients with PTC have a high rate of regional metastasis, with clinically apparent lymph node involvement 
in $15 \%$ to $50 \%$ and occult metastasis in $40 \%$ to $90 \%{ }^{(2)}$.

Because lymph node metastasis has not been considered prognostic for poor survival, its management is controversial. However, cervical lymph node recurrences have been reported in up to $31 \%$ of patients $^{(3)}$.

The incidence of local recurrence most likely is reduced by lymph node dissection, but neck dissection has been associated with significant morbidity, indicating the importance of better selection of patients PTC who would benefit from compartmentoriented lymph node dissection ${ }^{(4)}$.

Small, macroscopic metastatic lymph nodes in the lateral neck that were missed by palpation alone have been detected by high resolution cervical ultra sonography (US). However, US has a lower rate of detection of metastatic lymph nodes in the central compartment. Subclinical lymph node metastases can be detected by biopsy of the sentinel lymph node (SLN), which is defined as the first lymph node draining a regional lymphatic basin from a primary tumor ${ }^{(5)}$.

SLN sampling may avoid unnecessary lymph node dissection in patients with PTC who do not demonstrate spread to regional lymph nodes. complete central neck lymph node dissection, including patients whose lymph nodes did not stain, may be required to establish the utility of SLN sampling ${ }^{(6)}$.

Therefore, we prospectively assessed the usefulness of SLN biopsy for the detection of central lymph node metastasis in patients with PTC who didn't have suspected cervical lymphadenopathy.

\section{AIM OF THE WORK:}

The aim of this study wasto evaluate the accuracy of using sentinel lymph node biopsy as a marker to identify occult metastases early and could avoid the morbidity associated with routine and unnecessary lymph node dissection.

\section{PATIENTS AND METHODS:}

The present study was a prospective study that was conducted in Ain Shams University Hospitals in Egypt, and included sixty (60) patients who had papillary thyroid carcinoma. The patients underwent sentinel lymph node biopsy with total thyroidectomy during the period between 1stof December 2016 and $1^{\text {st }}$ of December 2018. Informed consent was obtained from each patient. All cases were operated by or under supervision of a consultant surgeon.

\section{Inclusion Criteria:}

Any patient with papillary thyroid carcinoma diagnosed by F.N.A regardless age, sex, size of tumor, multifocality or bilaterality was included in this study.

\section{Exclusion Criteria:}

Patients with high risk of anesthesia (ASA IV or V) not candidate for surgery.

Any patient had a history of thyroid or neck surgery for nonthyroidal head and neck cancers.

Palpable or US-detected lymph node involvement.

$>$ Other types of thyroid malignancies.

\section{Pre-operative assessment:}

Full clinical history and clinical examination (general and local), routine pre-operative blood tests (complete blood picture, coagulation profile, liver and kidney functions tests), plain chest X-ray, ECG and echocardiography (if indicated), thyroid function test (T.S.H, free T3 \& free T4), neck ultrasound, thyroglobulin level as a baseline, F.N.A from thyroid nodule and routine vocal cord assessment. 


\section{Pre-operative preparation:}

Control of any coexisting medical disease especially coexisting chest diseases. All patients signed the informed consent and dexamethasone was given with induction of anesthesia.

\section{Surgical procedure and detection of SLN:}

Under general anesthesia, a standard transverse low-collar skin incision was made; dissection of upper and lower skin flaps was performed and the strap muscles were retracted laterally. Before mobilization, the thyroid gland and the ipsilateral jugular vein were exposed. By using a 30-gauge insulin needle, $0.2-1 \mathrm{~mL}$ of $2 \%$ methylene blue (not more so as not to cause staining of surrounding tissues and this masks lymphatics and becomes misleading) was injected into the tumor (intratumoral); in a large nodule, injection was carried out at 3, 6, 9, 12 o'clock. Within few seconds, the stained lymphatic channels over the thyroid gland were traced to the central compartment and were followed up to the first blue-stained lymph node, which was called the "SLN" and the other LNs in the central compartment were called "non"SLN. In bilateral nodules the procedure was done on both sides. If there were no stained nodes, the first node closest to the afferent stained lymphatic vessel was considered the SLN. After SLN biopsy, total thyroidectomy with dissection of the central neck compartment and clearance of pretracheal and paratracheal nodes was routinely performed. During the operation the parathyroid glands were preserved on their arterial supply and both recurrent laryngeal nerves were identified and followed to the entrance into the larynx. The SLNs were harvested and sent for paraffin sections.
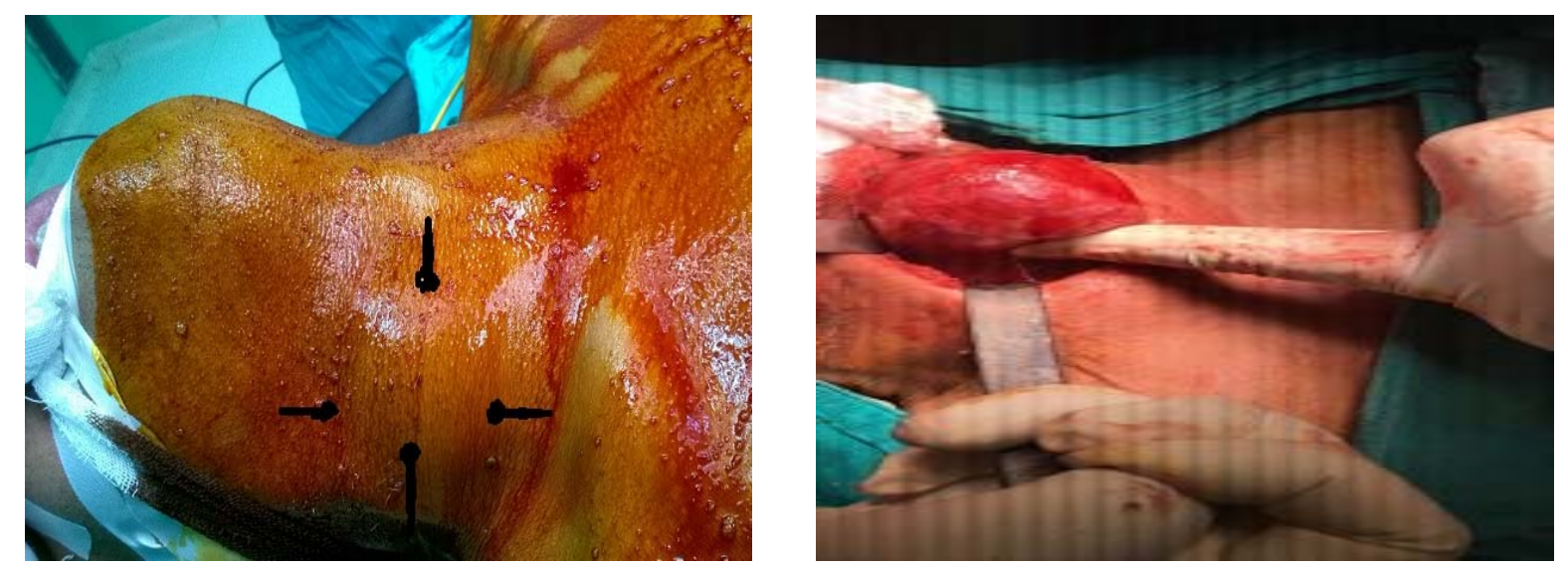

Fig (1): (A): Male patient with right thyroid nodule (arrows) (B): Intra-operative the nodule before dissection of the lobe so as not to cut lymphatics. 


\section{Ayman Abd-Allah Abd-Rabu, et al.,}
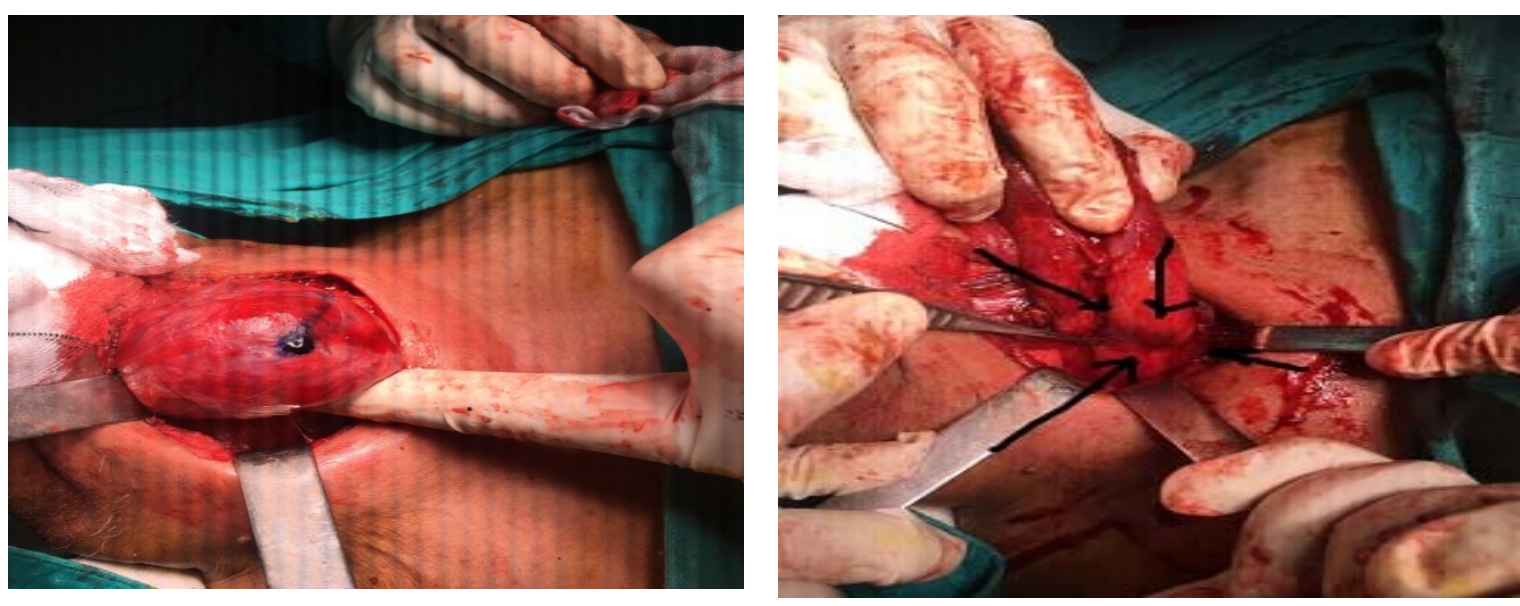

Fig (2): (A):Injection of methelyne blue intratumoral (B): Lymphatics arising from the nodule and passing laterally.
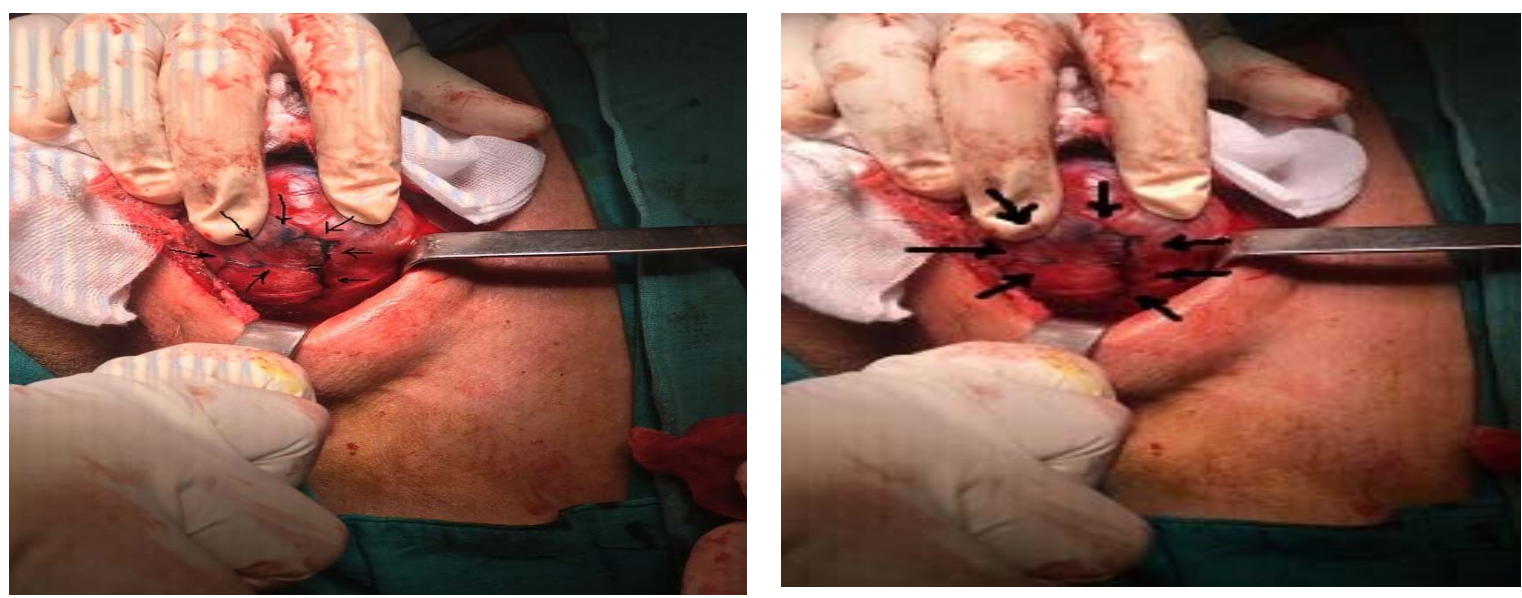

Fig (3): (A): Another view of lymphatics passing laterally to the paratracheal LNs (B): Arrows points to the sentinel LNs.
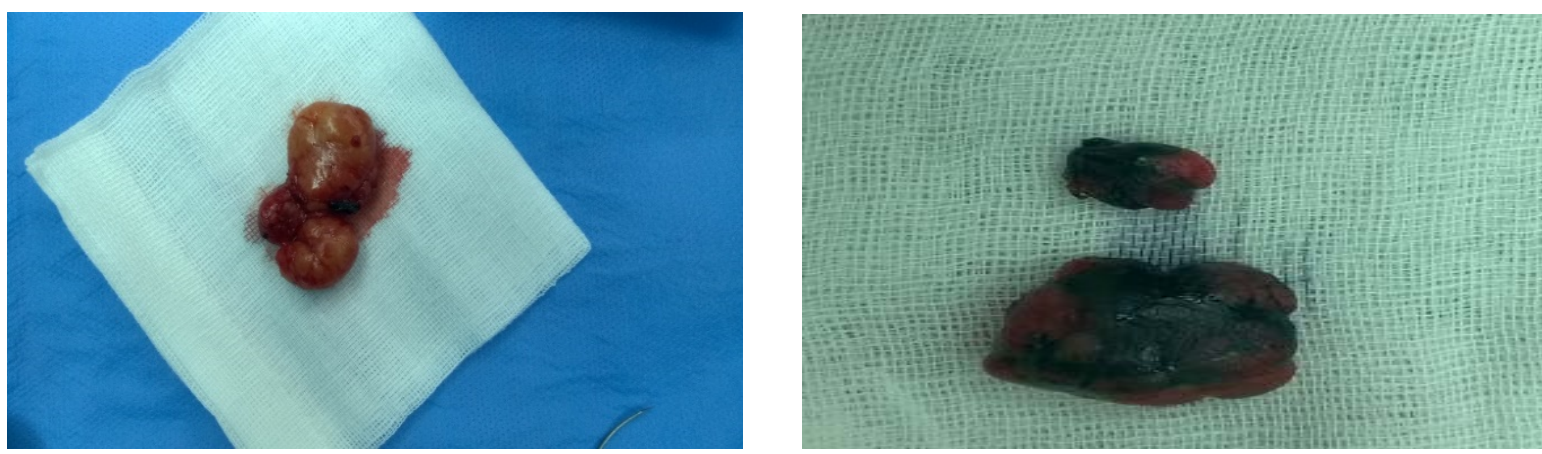

Fig (4): (A)\&(B): LNs after extraction. 


\section{Post-operative follow up:}

Suppression dose of thyroxine therapy will be administered to all patients. All patients will be followed regularly by clinical examination and neck US. Neck \& whole-body radioiodine scanning, and serum thyroglobulin measurements to be on regular intervals 1,2,3 months.

\section{Statistical analysis:}

Data were collected tabulated and statically analyzed. Analysis of data was done using SPSS (statistical program for social science version 21) as follows: Descriptive statistics were used to analyze patient and tumor characteristics. Description of quantitative variables was done as number and percentage. Accuracy, sensitivity, specificity, and positive and negative predictive values of the SLN were calculated.

\section{RESULTS:}

The characteristics of patients and tumor are summarized in Table 1 . There were a total of 60 patients, of whom 44 were women and 16 were men, and their age ranged from 23 to 60 years, the tumor size ranged from 0,5 to $4 \mathrm{~cm}$; 38 patients had left lobe tumor and 22 patients had right lobe tumor.

Table 1 Patient characteristics and presentations

\begin{tabular}{|l|l|}
\hline Number of patients & 60 \\
\hline Age & $23-60$ \\
\hline Females & 44 \\
\hline Males & 16 \\
\hline Tumor size (cm) & $0,5-4$ \\
\hline Left Lobe Tumor & 38 \\
\hline Right Lobe Tumor & 22 \\
\hline Hospitalization (days) & $2-5$ \\
\hline
\end{tabular}

The locations of identified SLN are listed in Table 2. Of the SLNs detected, 25 patients $(41,6 \%)$ were located in the ipsilateral paratracheal group, followed by 7 patients $(11,6 \%)$ in the pretracheal group, 7 patients $(11,6 \%)$ in the prelaryngeal group, and 5 patients $(8,3 \%)$ in the contralateral paratracheal group

Table 2 Location of identified SLNs in the central compartment

\begin{tabular}{|l|l|}
\hline Subsite & Detected SLN [N (\%)] \\
\hline Ipsilateral paratracheal group & $25(41,6 \%)$ \\
\hline Pretracheal group & $7(11,6 \%)$ \\
\hline Prelaryngeal group & $7(11,6 \%)$ \\
\hline Contralateral paratracheal group & $5(8,3 \%)$ \\
\hline Total & $44(73,3 \%)$ \\
\hline
\end{tabular}

Characteristics of sentinel lymph node (SLN) and non-sentinel lymph node (NSLN) are listed in Table 3 (a,b)

Table (3a): Characteristics of sentinel lymph node (SLN) and non-sentinel lymph node (NSLN):

\begin{tabular}{|l|l|l|}
\hline & \multicolumn{1}{|c|}{ SLN } & \multicolumn{1}{c|}{ Non SLN } \\
\hline & \multicolumn{1}{|c|}{ No \& Percentage } & \multicolumn{1}{c|}{ No \& Percentage } \\
\hline Identification rate of SLN & $44 / 60(73.3 \%)$ & $56 / 60(93.3 \%)$ \\
\hline Number of LN detected(range ,mean \pm SD) & $0-6\left(2.8+\_1.9\right)$ & $0-9\left(2.4+\_1.3\right)$ \\
\hline Size of SLN detected (range cm) & $0.1-1.6 \mathrm{~cm}$ & $0.1-1.5 \mathrm{~cm}$ \\
\hline LN metastasis found positive & $38 / 60(63,3 \%)$ & $31 / 60(51.7 \%)$ \\
\hline LN metastasis found negative & $22 / 60(36,7 \%)$ & $29 / 60(48.3 \%)$ \\
\hline
\end{tabular}


Table (3b):

- Total number of cases +ve SNL \& NSLN: (total cases with Actual LN disease present)

- Total number of cases -ve SNL\&NSLN: (total cases actual LN disease absent)

- Number of cases -ve SNL \& -ve NSLN (true -ve)

- Number of cases -ve SNL \& +ve NSLN (false -ve)

- Number of cases +ve SNL \& -ve NSLN (false +ve)

\begin{tabular}{|l|l|l|l|}
\hline & Disease present & Disease absent & Total \\
\hline SLN +ve & True +ve (a) & False +ve (b) & a+b \\
\hline SLN -ve & False -ve (c) & True -ve (d) & c+d \\
\hline Total & $\mathrm{a}+\mathrm{c}$ & $\mathrm{b}+\mathrm{d}$ & $\mathrm{a}+\mathrm{b}+\mathrm{c}+\mathrm{d}$ \\
\hline
\end{tabular}

Sensitivity $=\mathrm{a} /(\mathrm{a}+\mathrm{c})$, Specificity $=\mathrm{d} /(\mathrm{b}+\mathrm{d})$, Accuracy $=\mathrm{a}+\mathrm{c} /(\mathrm{a}+\mathrm{b}+\mathrm{c}+\mathrm{d}), \cdot$ Negative predictive value $(\mathrm{NPV})=\mathrm{d} /(\mathrm{c}+\mathrm{d}), \cdot$ Positive predictive value $(\mathrm{PPV})=\mathrm{a} /(\mathrm{a}+\mathrm{b})$

Table (4): Accuracy of the SLN:

\begin{tabular}{|l|l|l|}
\hline & NO & Percentage \\
\hline - Sensitivity & $38 / 44$ & $86.7 \%$ \\
\hline - Specificity & $16 / 16$ & $100 \%$ \\
\hline - Negative predictive value & $16 / 22$ & $72.7 \%$ \\
\hline - Positive predictive value & $38 / 38$ & $100 \%$ \\
\hline - Accuracy & $44 / 60$ & $73.3 \%$ \\
\hline
\end{tabular}

Table 5 Complications of SLNB in PTC

\begin{tabular}{|l|l|}
\hline Complications & Number of patients (\%) \\
\hline Blue dye & $0(0)$ \\
\hline RLN paralysis & \\
Temporary & $2(3,3)$ \\
Permanent & $0(0)$ \\
\hline Hypoparathyroidism & \\
Temporary symptomatic & $3(5)$ \\
Temporary laboratory & $6(10)$ \\
\hline Mortality & $0(0)$ \\
\hline
\end{tabular}

PTC, papillary thyroid carcinoma; RLN, recurrent laryngeal nerve, SLNB, sentinel lymph node biopsy

\section{DISCUSSION:}

The prognosis of patients with thyroid carcinoma associated with cervical lymph node metastasis is poor compared to those without LN metastasis. Factors associated with persistent disease or recurrent papillary thyroid carcinoma are extranodal invasion, lymph node metastasis, a tumor size $>3 \mathrm{~cm}$, extra-capsular extension, and the locations of metastatic lymph nodes with central compartment lymph nodes being the most frequently involved site . So, complete surgical removal of lymph nodes in the central compartment during thyroid cancer surgery is important to decrease recurrence and mortality ${ }^{(7)}$.

The SLN is defined as the first lymph node in aregional lymphatic basin that drains a primary tumor. Conceptually, if lymphatic drainage is to occur in astepwise manner, then the SLN should reflect the pathologic status of the remaining lymph no debasin. SLNB, moreover, should beneficially detectearly, subclinical metastasis. In the last two decades, SLN biopsy has gained significant consensus as the standard of management for identifying regional lymphatic spread in melanoma and breast cancer $^{(8,9)}$.

Our study was conducted on 60 patients who had at a Papillary Thyroid carcinoma. The size of malignant nodules ranged from $0.5-4 \mathrm{~cm}$.Sizes of most nodules ranged between one and three $\mathrm{cm}$ and were present 
more in the left lobe (63.3\%) and to lesser extent in the right lobe (36.6\%).

In our patients, we used methylene blue injected inside the thyroid nodule (intratumoral) to identify the sentinel LN. Other methods for identifying SLN in differentiated thyroid carcinoma include intra-operative gamma probe and lymphoscintigraphy .

Several studies that used radioisotopes and intraoperative gamma probes for SLNB have reported higher detection rates (96$100 \%$ ) than the blue dye technique, although there was no marked difference in sensitivity and specificity ${ }^{(10)}$.

In our patients, sentinel LNs were identified in $46 / 60$ patients of malignant cases (accuracy). Number of LNs ranged from 0-6 (12 \pm 1.9$)$ and size ranged from 0.1$1.6 \mathrm{~cm}$. 38/60 (63,3\%) SLN were positive for metastasis and 22/60 (36.7\%) were negative for metastasis. Non sentinel LNs were extracted via the central compartment dissection. 31/60 was found positive for metastasis and 29/60 were found negative.

Another Study reported 33 patients who underwent SLN biopsy using a dye technique. Sentinel nodes were identified in $16 / 17$ patients $(94 \%)^{(11)}$

A study that carried out SLN biopsy on 40 patients. Sentinel node(s) were identified in 10 of 12 patients (83\%). The FNR was $20 \%$. In this study, the detection rate of SLN with methylene blue dye was $84 \%$. This is within the range (83-94\%) of prior studies that used the blue dye technique ${ }^{(12)}$.

From our results SLN had sensitivity, specificity, negative predictive value, positive predictive value and accuracy of $86.7 \%, \quad 100 \%, \quad 72.7 \%, \quad 100 \%, \quad 73.3 \%$ respectively.

Takami et al in $2003^{(13)}$ found that SLN have a sensitivity of $87.55 \%$ (35/40), specificity of $100 \%$ (23/23), positive predictive value of $100 \%$ (35/35), negative predictive value of $82.1 \% \quad(23-28)$ and accuracy of $92.1 \%$ (58/63).

Dzokc in $2006^{(\mathbf{1 4})}$ found the sensitivity of SLN to be $77.7 \%$, specificity $100 \%$, negative predictive value $94 \%$ positive predictive value $100 \%$ and overall accuracy 95\%.

In our study postoperatively, temporary symptomatic hypocalcemia developed in 3 of 60 patients (5\%), and laboratory hypocalcemia developed in 6 patients(10\%); no patient had permanent hypocalcemia. Two patients had postoperative temporary paralysis of the unilateral vocal cord, and no permanent paralysis was observed. mortality was not recorded.

Postoperative complication rate, including recurrent laryngeal nerve injury, hypoparathyroidism, and postoperative hematoma, was not different from previous studies of thyroidectomy. Hence, the addition of SLNB to thyroidectomy looks feasible and safe ${ }^{(15)}$.

\section{Conclsuion:}

From our study, we concluded that in patients having Papillary carcinoma of thyroid without evidence of macroscopic clinical or US-detected lymph node metastasis, sentinel lymph node biopsy can discover occult metastases with high accuracy and no morbidity. Improvement of lymph node metastatic identification using SLN biopsy may help select patients who would benefit from central compartment neck dissection and sparing those who do not need.

\section{REFRENCES:}

1. Dixon E, McKinnon JG and Pasieka JL. Feasibility of sentinel lymph node biopsy and lymphatic mapping in nodular thyroid neoplasms. World J Surg. 2010;24:13961401.

2. Dzodic R, Markovic I, Inic $M$, et al. Sentinel lymph node biopsy may be used to 
support the decision to perform modified radical neck dissection in differentiated thyroid carcinoma. World J Surg. 2016; 30:841-846.

3. Fukui Y, Yamakawa T, Taniki T, Numoto S, Miki H and Monden Y. Sentinel lymph node biopsy in patients with papillary thyroid carcinoma. Cancer. 2011; 92:28682674.

4. Noguchi M, Kinami S, Kinoshita K, et al. Risk of bilateral cervical lymph node metastases in papillary thyroid cancer. J Surg Oncol. 2003;52:155-159.

5. Pelizzo MR, Boschin IM, Toniato A, et al. The sentinel node procedure with Patent Blue $\mathrm{V}$ dye in the surgical treatment of papillary thyroid carcinoma. Acta Otolaryngol. 2011; 121:421-424.

6. Singer PA, Cooper DS, Daniels GH, et al. Treatment guidelines for patients with thyroid nodules and well-differentiated thyroid cancer. American Thyroid Association. Arch Intern Med. 2006;156:2165-2172.

7. Lundgren CI, Hall P, Dickman PW, Zedenius J: Clinically significant prognostic factors for differentiated thyroid carcinoma: a population-based, nested case-control study. Cancer 2006; 106: 524-531.

8. Pelizzo MR, Rubello D, Boschin IM, Piotto A, Paggetta C, Toniato A, et al.: Contribution of SLN investigation with 99mTc-nanocolloid in clinical staging of thyroid cancer: Technical feasibility. Eur J Nucl Med Mol Imaging 2007; 34: 934938.
9. Balasubramanian SP, Harrison BJ. Systematic review and meta-analysis of sentinel node biopsy in thyroid cancer. $\mathrm{Br} \mathrm{J}$ Surg 2011; 98:334-344.

10. Ramin S, Azar FP, Malihe H. Methylene blue as the safest blue dye for sentinel node mapping: emphasis on anaphylaxis reaction. Acta Oncol 2011; 50:729-731.

11. Haigh PI, Giuliano AE. Sentinel lymph node dissection for thyroid malignancy. Recent Results Cancer Res 2000; 157:201205.

12. Dixon E, McKinnon J, Pasieka J. Feasibility of sentinel node biopsy and lymphatic mapping in nodular thyroid neoplasms. World J Surg 2000; 24:1396-1401.

13. Takami H, Sasaki K, Ikeda Y, Tajima G, Kameyama K: Detection of Sentinel Lymph Nodes in Patients with Papillary Thyroid Cancer. ASIAN JOURNAL OF SURGERY 2003; Jul; 26(3):145-8.

14. Dzodic R: Sentinel Lymph Node Biopsy May Be Used to Support the Decision to Perform Modified Radical Neck Dissection in Differentiated Thyroid Carcinoma World J Surg (2006) 30: 841-846.

15. Cavicchi O, Piccin O, Caliceti U, DeCataldis A, Pasquali R, Ceroni A R. Transient hypoparathyroidism following thyroidectomy: a prospective study and multivariate analysis of 604 consecutive patients. Otolaryngol Head Neck Surg 2007; 137:654-658. 